\title{
N umerical Solution for $M$ ixed Convection Heat Transfer from a Vertical Heated Plate Embedded in a Sparsely Packed Porous Medium
}

\author{
N. Nalinakshi,* P.A. Dinesh,† I.S. Shivakumarał and D.V. \\ Chandrashekar§
}

\section{Abstract}

An improved numerical study on mixed convection from a heated vertical plate embedded in a Newtonian fluid saturated sparsely packed porous medium is undertaken by considering the variation of permeability, porosity and thermal conductivity. The boundary layer flow in the porous medium is governed by Lapwood - Forchheimer - Brinkman extended Darcy model. Similarity transformations are employed and the resulting ordinary differential equations are solved numerically by using shooting algorithm with Runge - Kutta - Fehlberg integration scheme to obtain velocity and temperature distributions. Besides, skin friction and Nusselt number are also computed for various physical parameters governing the problem under consideration. It is found that the inertial parameter has a significant influence in decreasing the flow field, whereas its influence is reversed on the rate of heat transfer for all values of permeability considered. Further, the obtained results under the limiting conditions were found to be in good agreement with the existing ones.

\footnotetext{
* A tria Institute of Technology, Bangalore 560 024; nalinigcm@yahoo.co.in † M. S. Ramaiah Institute of Technology, Bangalore 560 054; dineshdpa@msrit.edu ‡ UGC - CAS in Fluid Mechanics, BangaloreUniversity

$\S$ Vivekananda Institute of Technology, Bangalore 560 074; dvchandru@yahoo.com
} 
Keywords: Heat Transfer, Mixed Convection Heat Transfer, Newtonian fluid, Porous Medium

\section{Introduction}

In recent years, considerable attention has been evinced on the study of boundary layer flow behavior and heat transfer characteristics of N ewtonian fluid past a vertical plate embedded in a fluid saturated porous medium because of its extensive applications in engineering processes, especially in the enhanced recovery of petroleum resources and packed bed reactors. Considerable amount of interest has also been devoted towards the study of transport properties in porous media subject to heat transfer which are characterized by highly non-linear coupled partial differential equations. The problem of free convection heat transfer from a vertical plate embedded in a fluid saturated porous medium is studied by Cheng and Minkowyc (1977), who have obtained the similarity solutions for the problem considered. Cheng (1978) has provided an extensive review of early works on free convection in porous media. Nakayama and Koyama (1987) have obtained the similarity solution for the problem of free convection in the boundary layer adjacent to a vertical plate immersed in a thermally stratified porous medium. The mixed convection boundary layer flow on an impermeable vertical surface embedded in a saturated porous medium has been treated by Merkin (1980). Hung and Chen (1997) have studied non-Darcy free convection in a thermally stratified fluid saturated porous medium along a vertical plate with variable heat flux. Hsieh et al.(1993) have obtained non-similar solution for combined convection from vertical plates in porous media with variable surface temperatures or heat flux. Recently, Nield and Bejan (1999) have given an excellent summary of free convection flow in porous media.

Several investigators have considered the non - Darcian model in the recent past to study the convection and heat transfer rates on bodies embedded in a porous medium for Newtonian fluids. Kumari et al (1990) have investigated the non-Darcian effects on forced convection heat transfer over a flat plate in a highly porous medium. Chen and $\mathrm{Ho}(1988)$ have studied the effects of flow 
inertia on vertical, natural convection in saturated, porous media. Hong et al.(1987) have studied analytically the non-Darcian effects on a vertical plate natural convection in porous media. They used a combination of Rayleigh and Darcy numbers to describe the inertia and boundary terms and obtained similar solutions. They found that these effects decrease the velocity and reduce the heat transfer rate. Hassanien et al (1998) studied the effects of thermal stratification on non-Darcy mixed convection from a vertical flat plate embedded in a porous medium. Plumb and Huenefeld (1981) investigated on non-Darcy natural convection from vertical isothermal surfaces in saturated porous media. Lai and Kulacki $(1987,1991)$ used both Darcy and non-Darcy natural convection from vertical isothermal surfaces in saturated porous media. Bejan and Poulikakos (1984) used Forchheimer's model to study vertical boundary layer natural convection in a porous medium.

Shwartz and Smith (1953), Benenati and Brosilow (1962) have shown that the permeability of a porous medium varies due to the variation of porosity from the wall to the interior of the porous medium. Chandrashekar and Namboodire (1985) have shown the effectiveness of variable permeability of the porous medium on velocity distribution and heat transfer. Recently, Mohammadein and El-Shaer (2004) have studied combined free and forced convective flow past a semi-infinite vertical plate embedded in a porous medium incorporating the variable permeability. Nonetheless, the inertia effects become important in a sparsely packed porous medium and hence their effect on free convection problems needs to be investigated.

The aim of the present investigation is, therefore, to study systematically the effect of inertial terms on combined free and forced convective heat transfer past a semi-infinite vertical plate embedded in a saturated porous medium with variable permeability, porosity and thermal conductivity. In this analysis coupled non-linear partial differential equations, governing the problem, are first reduced by a similarity transformation to the ordinary differential equations and then the resultant boundary value problem is converted into the system of five simultaneous equations of first-order for five unknowns. Then these equations are solved numerically by shooting technique with Runge-Kutta- 
Fehlberg method to obtain horizontal velocity and temperature profiles for various values of physical parameters. The results obtained from the present numerical computation under limiting conditions agree well with the existing ones and thus verifies the accuracy of the method used.

\section{M athematical Formulation}

We consider a semi-infinite vertical heated plate embedded in a sparsely packed Newtonian fluid saturated porous medium of variable porosity, permeability and thermal conductivity. The $x$ coordinate is measured along the plate from its leading edge, and $y$-coordinate normal to it. Let $U_{o}$ be the velocity of the fluid in the upward direction and the gravitational field, $g$, is acting in the downward direction. The plate is maintained at a uniform temperature $T_{w}$ which is always greater than the free stream values existing far from the plate (i.e., $T_{w}>T_{\infty}$ ). The boundary layer equations governing the conservation of mass, momentum and energy can be written in the following form:

$$
\begin{gathered}
\frac{\partial u}{\partial x}+\frac{\partial v}{\partial y}=0 \\
\rho\left(u \frac{\partial u}{\partial x}+v \frac{\partial u}{\partial y}\right)=\rho g \beta\left(T-T_{\infty}\right)+\bar{\mu} \frac{\partial^{2} u}{\partial y^{2}}+\frac{\mu \varepsilon(y)}{k(y)}\left(U_{o}-u\right)+\frac{F \varepsilon^{2}(y)}{\sqrt{k(y)}}\left(U_{o}^{2}-u^{2}\right)(2) \\
\left(u \frac{\partial T}{\partial x}+v \frac{\partial T}{\partial y}\right)=\frac{\partial}{\partial y}\left(\alpha(y) \frac{\partial T}{\partial y}\right)+\frac{\bar{\mu}}{\rho C_{p}}\left(\frac{\partial u}{\partial y}\right)^{2}
\end{gathered}
$$

where, $u$ and $v$ are the velocity components along the $x$ and $y$ directions respectively, $\mathrm{T}$ is the temperature of the fluid, $\rho$ is the fluid density, $\mathrm{g}$ is the acceleration due to gravity, $\bar{\mu}$ is the effective viscosity of the fluid, $\mu$ is the fluid viscosity, $k(y)$ is the variable permeability of the porous medium, $\varepsilon(y)$ is the porosity of the saturated porous medium, $\alpha(y)$ is the variable effective thermal diffusivity of the medium, $\mathrm{F}$ is the empirical constant of the second 
M apana J Sci, 10, 2(2011) Mixed Convetion HedTransfer fromaVertical Heeted Plate

- order resistance term due to inertial effect, $C_{p}$ is the specific heat at constant pressure, $\beta$ is the coefficient of volume expansion and $T_{\infty}$ is the ambient temperature.

The above governing equations need to be solved subject to the following boundary conditions on velocity and temperature fields:

$$
\begin{array}{cr}
u=0, \quad v=0, \quad T=T_{w} & \text { at } y=0 \\
u=U_{o}, \quad v=0, \quad T=T_{\infty} & \text { as } y \rightarrow \infty
\end{array}
$$

We now introduce the following dimensionless variables $f$ and $\theta$ as well as the similarity variable $\eta$

[Hady et al.(1996), M ohammadein and El-Shaer (2004)]:

$$
\eta=\left(\frac{y}{x}\right)\left(\frac{U_{o} x}{v}\right)^{1 / 2}, \psi=\sqrt{v U_{o} x} f(\eta), \quad \theta=\frac{T-T_{\infty}}{T_{w}-T_{\infty}}
$$

where, a prime represents differentiation with respect to $\eta$ and $T_{w}$ is the plate temperature.

In equation (6) the stream function $\psi(x, y)$ is defined by $u=\frac{\partial \psi}{\partial y}$, $v=-\frac{\partial \psi}{\partial x}$, such that the continuity equation (1) is satisfied automatically and the velocity components are given by

$$
u=U_{o} f^{\prime}(\eta), \quad v=-\frac{1}{2} \sqrt{\frac{v U_{o}}{x}}\left(f(\eta)-\eta f^{\prime}(\eta)\right)
$$

Following Chandrashekhara and Namboodiri (1985), the variable permeability $k(\eta)$, the variable porosity $\varepsilon(\eta)$ and variable effective thermal diffusivity $\alpha(\eta)$ are given by

$$
\begin{gathered}
k(\eta)=k_{o}\left(1+d e^{-\eta}\right) \\
\varepsilon(\eta)=\varepsilon_{o}\left(1+d^{*} e^{-\eta}\right) \\
\alpha(\eta)=\alpha_{o}\left[\varepsilon_{o}\left(1+d^{*} e^{-\eta}\right)+\sigma^{*}\left\{1-\varepsilon_{o}\left(1+d^{*} e^{-\eta}\right)\right\}\right]
\end{gathered}
$$


Where $k_{o}, \varepsilon_{o}$ and $\alpha_{o}$ are the permeability, porosity and diffusivity at the edge of the boundary layer respectively, $\sigma^{*}$ is the ratio of the thermal conductivity of solid to the conductivity of the fluid, $d$ and $d^{*}$ are treated as constants having values 3.0 and 1.5 respectively.

Substituting (6) and (7) in Equations (2) and (3), we get the following transformed equations:

$$
\begin{gathered}
f^{\prime \prime \prime}+\frac{1}{2} f f^{\prime \prime}+\frac{G r}{\operatorname{Re}^{2}} \theta+\frac{\alpha^{*}\left(1+d^{*} e^{-\eta}\right)}{\sigma \operatorname{Re}\left(1+d e^{-\eta}\right)}\left(1-f^{\prime}\right)+\frac{\beta^{*}\left(1+d^{*} e^{-\eta}\right)^{2}}{\left(1+d e^{-\eta}\right)^{1 / 2}}\left(1-f^{\prime 2}\right)=0 \\
\theta^{\prime \prime}=-\frac{(1 / 2) \operatorname{Pr} \theta^{\prime} f+\operatorname{Pr} E f^{\prime \prime 2}+\varepsilon_{o} d^{*} e^{-\eta}\left(\sigma^{*}-1\right) \theta^{\prime}}{\varepsilon_{o}+\sigma^{*}\left(1-\varepsilon_{o}\right)+\varepsilon_{o} d^{*} e^{-\eta}\left(1-\sigma^{*}\right)}
\end{gathered}
$$

where, $\beta^{*}=F \varepsilon_{o}^{2} x / k_{o}^{1 / 2}$ is the local inertial parameter, $\operatorname{Pr}=\bar{\mu} / \rho \alpha_{o}$ is the Prandtl number, $\alpha^{*}=\mu / \bar{\mu}$ is the ratio of viscosities, $E=U_{o}^{2} / C_{p}\left(T_{w}-T_{\infty}\right) \quad$ is the Eckert number, $\sigma=k_{o} / x^{2} \varepsilon_{o}$ is the local permeability parameter, $\operatorname{Re}=U_{o} x / v$ is the local Reynolds number and $G r=g \beta\left(T_{w}-T_{\infty}\right) x^{3} / v^{2}$ is the local Grashof number.

The transformed boundary conditions are:

$$
\begin{aligned}
& f=0, \quad f^{\prime}=0, \quad \theta=1 \quad \text { at } \eta=0 \\
& f^{\prime}=1, \quad \theta=0 \quad \text { as } \eta \rightarrow \infty
\end{aligned}
$$

Once the velocity and temperature distributions are known, the skin friction and the rate of heat transfer can be calculated respectively by

$$
\tau=-f^{\prime \prime}(0) / \sqrt{\operatorname{Re}} \quad \text { and } \quad N u=-\sqrt{\operatorname{Re}} \theta^{\prime}(0)
$$

where $\tau$ is theskin friction and $\mathrm{Nu}$ is the Nusselt number.

\section{3. $N$ umerical M ethod}

Equations (11) and (12) constitute a highly non-linear coupled boundary value problem (BVP) of third and second order respectively. An improved numerical scheme involving shooting 
technique with Runge-Kutta-Fehlberg method is developed to solve the resulting nonlinear BVP. Thus, the coupled nonlinear boundary value problem of third-order in $\mathrm{f}$ and second-order in $\theta$ has been reduced to a system of five simultaneous equations of first-order for five unknowns as follows (Vajravelu, 2001):

$$
\begin{gathered}
f_{1}^{\prime}=f_{2}, \quad f_{2}^{\prime}=f_{3}, \\
f_{3}^{\prime}=-\frac{1}{2} f_{1} f_{3}-\frac{G r}{\operatorname{Re}^{2}} f_{4}-\frac{\alpha^{*}\left(1+d^{*} e^{-\eta}\right)}{\sigma \operatorname{Re}\left(1+d e^{-\eta}\right)}\left(1-f_{2}\right)-\frac{\beta^{*}\left(1+d^{*} e^{-\eta}\right)}{\left(1+d e^{-\eta}\right)^{1 / 2}}\left(1-f_{2}^{2}\right), \\
f_{4}^{\prime}=f_{5}, \quad f_{5}^{\prime}=-\frac{(1 / 2) \operatorname{Pr} f_{1} f_{5}+\operatorname{Pr} E f_{3}^{2}+\varepsilon_{o} d^{*} e^{-\eta}\left(\sigma^{*}-1\right) f_{5}}{\varepsilon_{o}+\sigma^{*}\left(1-\varepsilon_{o}\right)+\varepsilon_{o} d^{*} e^{-\eta}\left(1-\sigma^{*}\right)}
\end{gathered}
$$

where $f_{1}=f, f_{2}=f^{\prime}, f_{3}=f^{\prime \prime}, f_{4}=\theta, f_{5}=\theta^{\prime}$ and a prime denotes differentiation with respect to $\eta$.

The boundary conditions now become

$$
\begin{array}{ll}
f_{1}=0, \quad f_{2}=0, \quad f_{4}=1 & \text { at } \quad \eta=0 \\
f_{2}=1, \quad f_{4}=0 & \text { as } \quad \eta \rightarrow \infty
\end{array}
$$

\section{Results and Discussion}

The system of first-order differential equations (17) - (19) are solved numerically using shooting technique with Runge-KuttaFehlberg method. In order to know the accuracy of the method used, computed values of $f^{\prime \prime}(0)$ and $\theta^{\prime}(0)$ were obtained for $\beta^{*}=0$ and compared with those obtained by Mohammadein and El-Shaer (2004) in Table 1 for the variable permeability $(d=3.0$, $d^{*}=1.5$ ) case and good agreement has been obtained with their results. The values tabulated in Table 1 are for $\varepsilon_{o}=0.4, E=0.1, \operatorname{Pr}=0.71$ with selected values of $G r / \operatorname{Re}^{2}, \sigma^{*}$ and $\sigma^{*} / \sigma \operatorname{Re}$. The slight deviation in the values may be due to the use of Runge-Kutta-Fehlberg method which has fifth order accuracy whereas, Mohammadein and El-Shaer (2004) have used fourthorder Runge-Kutta method which has only fourth order accuracy. 
Thus the present results are more accurate compared to their results.

Table 2 contains the computed values of $f^{\prime \prime}(0)$ and $-\theta^{\prime}(0)$ for the selected values of $\alpha^{*} / \sigma \operatorname{Re}$ and $G r / \operatorname{Re}^{2}$ and $\beta^{*}$ for uniform permeability (UP) and variable Permeability (VP) cases. From the table, it is observed that an increase in the value of $\beta^{*}$ is to increase the skin friction for all values of $\alpha^{*} / \sigma \operatorname{Re}, \sigma^{*}$ and $G r / \operatorname{Re}^{2}$ for both UP \& VP. Further, it is interesting to note the effect of $\sigma^{*}$ is to increase the skin friction whereas the rate of heat transfer decreases.

\begin{tabular}{|c|c|c|c|c|c|c|}
\hline \multirow{2}{*}{$\sigma^{*}$} & \multirow{2}{*}{$G r / \operatorname{Re}^{2}$} & \multirow{2}{*}{$\alpha^{*} / \sigma \operatorname{Re}$} & \multicolumn{2}{|c|}{ Present result } & \multicolumn{2}{|c|}{$\begin{array}{l}\text { Mohammadein } \\
\text { and El-Shaer }\end{array}$} \\
\hline & & & $f^{\prime \prime}(0)$ & $-\theta^{\prime}(0)$ & $f^{\prime \prime}(0)$ & $-\theta^{\prime}(0)$ \\
\hline \multirow{6}{*}{2.0} & \multirow{3}{*}{0.2} & 0.0 & 0.611321 & 0.381233 & 0.61215 & 0.38030 \\
\hline & & 0.1 & 0.667804 & 0.386090 & 0.64526 & 0.38281 \\
\hline & & 0.5 & 0.846341 & 0.417658 & 0.75527 & 0.38959 \\
\hline & \multirow{2}{*}{0.5} & 0.0 & 0.958156 & 0.403083 & 0.95816 & 0.40308 \\
\hline & & 0.1 & 0.987898 & 0.406430 & 0.97432 & 0.40325 \\
\hline & 2.0 & 0.0 & 2.415691 & 0.376339 & 2.31558 & 0.40376 \\
\hline \multirow{5}{*}{4.0} & \multirow{3}{*}{0.2} & 0.0 & 0.627031 & 0.504676 & 0.62705 & 0.50459 \\
\hline & & 0.1 & 0.681575 & 0.507192 & 0.65772 & 0.50664 \\
\hline & & 0.5 & 0.859094 & 0.519451 & 0.76231 & 0.51242 \\
\hline & \multirow[b]{2}{*}{0.5} & 0.0 & 0.993653 & 0.528672 & 0.99206 & 0.52979 \\
\hline & & 0.1 & 1.022091 & 0.528510 & 1.00403 & 0.52940 \\
\hline & & $c^{\prime \prime}$ & ricol & 0 & $1, \quad \beta$ & $=0.0$ for \\
\hline
\end{tabular}




\begin{tabular}{|c|c|c|c|c|c|c|c|}
\hline \multirow{2}{*}{$\alpha^{*} / \sigma \operatorname{Re}$} & \multirow{2}{*}{$\sigma^{*}$} & \multirow{2}{*}{$G r / \operatorname{Re}^{2}$} & \multirow{2}{*}{$\beta^{*}$} & \multicolumn{2}{|c|}{$f^{\prime \prime}(0)$} & \multicolumn{2}{|l|}{$-\theta^{\prime}(0)$} \\
\hline & & & & UP & VP & UP & VP \\
\hline \multirow{15}{*}{0.1} & \multirow{11}{*}{2.0} & \multirow{4}{*}{0.0} & 0.0 & 0.451835 & 0.421933 & 0.250491 & 0.363478 \\
\hline & & & 0.1 & 0.576676 & 0.564654 & 0.260377 & 0.379063 \\
\hline & & & 0.5 & 0.929158 & 0.963919 & 0.357305 & 0.504579 \\
\hline & & & 0.9 & 1.192470 & 1.284032 & 0.507254 & 0.796314 \\
\hline & & \multirow{4}{*}{0.1} & 0.0 & 0.584076 & 0.549309 & 0.260956 & 0.376262 \\
\hline & & & 0.1 & 0.690510 & 0.672864 & 0.267553 & 0.387279 \\
\hline & & & 0.5 & 1.007338 & 1.036579 & 0.288634 & 0.412960 \\
\hline & & & 0.9 & 1.245152 & 1.327359 & 0.506892 & 0.795579 \\
\hline & & \multirow{2}{*}{0.2} & 0.0 & 0.707080 & 0.667804 & 0.269021 & 0.386090 \\
\hline & & & 0.1 & 0.798848 & 0.776034 & 0.273416 & 0.393967 \\
\hline & & 2.0 & 0.1 & 2.368606 & 2.259598 & 0.291978 & 0.414071 \\
\hline & \multirow{4}{*}{4.0} & \multirow{2}{*}{0.0} & 0.1 & 0.576676 & 0.564841 & 0.217035 & 0.538623 \\
\hline & & & 0.5 & 0.929858 & 0.963919 & 0.351812 & 0.731295 \\
\hline & & \multirow{2}{*}{0.1} & 0.1 & 0.700834 & 0.671721 & 0.222808 & 0.545299 \\
\hline & & & 0.5 & 1.002954 & 1.028371 & 0.352147 & 0.731303 \\
\hline 0.5 & 2.0 & 0.2 & 0.0 & 0.937729 & 0.846341 & 0.336831 & 0.417658 \\
\hline
\end{tabular}

Table 2: Results for $f^{\prime \prime}(0)$ and $-\theta^{\prime}(0)$ for the selected values of $\alpha^{*} / \sigma \operatorname{Re}$ and $G r / \operatorname{Re}^{2}$ for $\operatorname{Pr}=0.71$ for uniform Permeability (UP) and Variable Permeability (VP) cases.

Figure 1 depicts the velocity distribution for various values of second order resistance for variable permeability (VP) and uniform permeability (UP) cases. It is observed that, increase in the value of inertial parameter $\beta^{*}$ leads to an increase in the velocity profile within the boundary layer, while for $\beta^{*}=0.5$, the velocity coincides for both UP and VP cases. It is also important to note that the boundary layer decreases with an increase in the value of inertial parameter. Thus, the non- Darcian term has a very significant effect on the velocity distribution. Figure 2 exhibits the variation of velocity profiles for various values of $\sigma^{*}$ for both UP and VP. It is clearly seen that the velocity profile increases with an increase in $\sigma^{*}$ which is effective only for UP but its effect diminishes for small values of $\sigma^{*}$. 


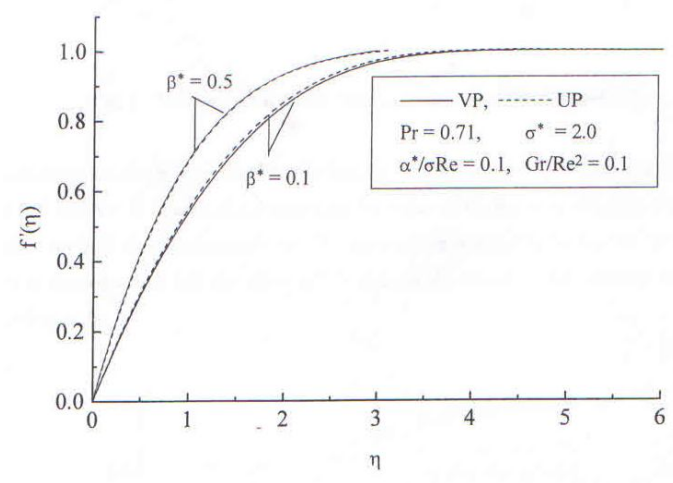

Figure 1: Velocity profiles for various values of second order resistance for VP and UP.

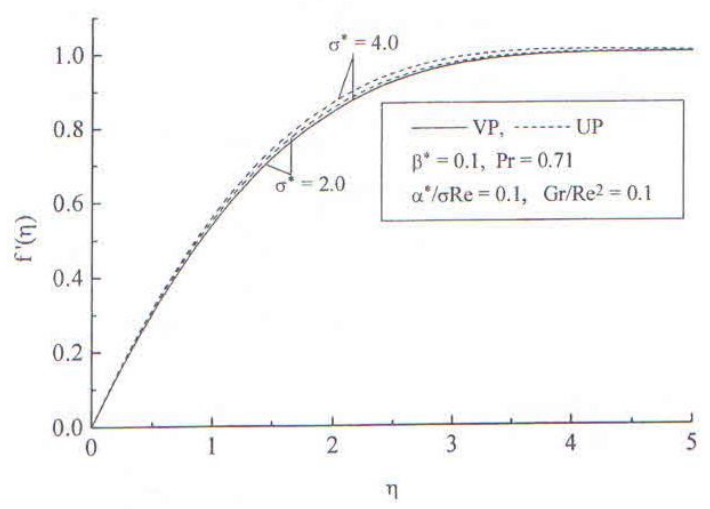

Figure 2: Velocity profiles for various values of $\sigma^{*}$ for VP and UP.

Figure 3 shows the variation of velocity distribution for three values of $\operatorname{Pr}=0.71,3$ and 10 for the case of VP. It is observed that the velocity profiles decrease as the Prandtl number increases 
M apana J Sci, 10, 2(2011) Mixed Convection Heet Tranfer fromaVertical Heeted Plate

which is very significant in the middle of boundary layer. Further, it is clear that the boundary layer decreases with decrease in the value of $\mathrm{Pr}$.

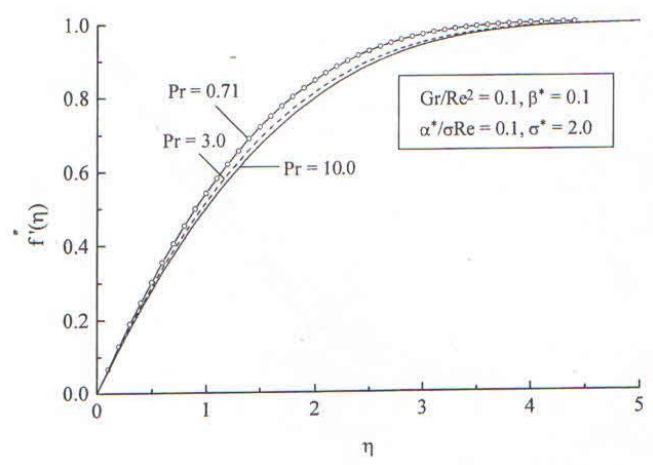

Figure 3: Variation of velocity distribution for various values of Prandt। number of VP

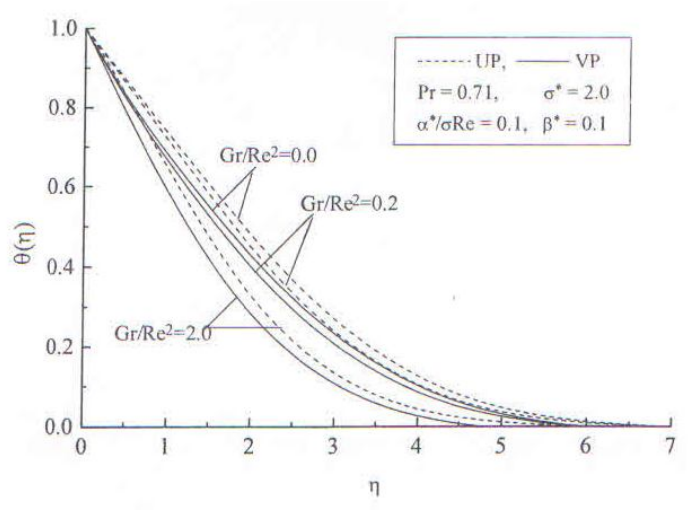

Figure 4: Temperature distribution for various values of $G r / \mathrm{Re}^{2}$ for VP and UP

Figure 4 depicts the temperature distribution for various values of the parameter $G r / \operatorname{Re}^{2}$ for the cases of UP and VP. It is seen that increase in the value of $G r / \operatorname{Re}^{2}$ is to decrease the temperature distribution for both the cases considered. The temperature is 
found to be less for VP as compared to UP. It is also observed that the effect of VP is more significant on temperature distribution for higher values of $\mathrm{Gr} / \mathrm{Re}^{2}$.

Figure 5 displays the distribution of temperature for various values of second order resistance $\beta^{*}$ for UP and VP cases. From this figure it is evident that the temperature profile decreases smoothly for $\beta^{*}=0.1$ within the boundary layer whereas for higher value of $\beta^{*}$ the temperature continuously decreases and this decrease is very rapid. This shows that the rate of cooling is much faster for higher values of second order resistance in both UP and VP cases.

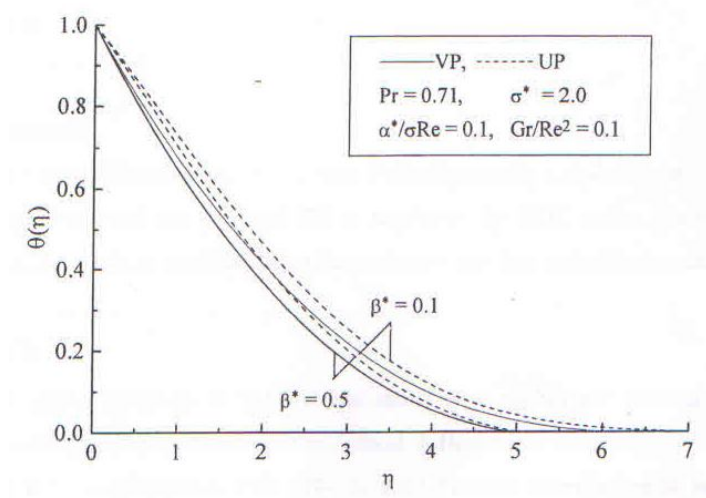

Figure 5: Temperature profiles for various values of second order resistance for VP and UP. 
M apana J Sci, 10, 2(2011) Mixed Convetion HedTransfer fromaVertical Heeted Plate

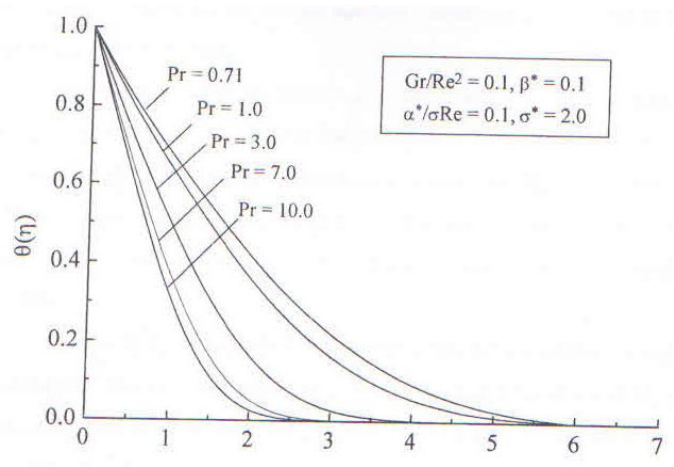

Figure 6: Variation of temperature distribution with $\eta$ for various values of Pr for VP.

A nother interesting feature which is observed from Figure 5 is that the boundary layer decreases with an increase in the value of inertial parameter and it is more so in the case of UP as compared to VP for both the values of $\beta^{*}$ considered. Figure 6 gives the variation of temperature distribution within the boundary layer for various values of $\mathrm{Pr}$ in the case of VP. The temperature profiles show a typical smooth decreasing pattern for $\operatorname{Pr}=0.71$ whereas, for higher values of Pr, the temperature continuously decreases at a steeper rate in the flow region and the boundary layer decreases with the increase in Pr.

\section{Acknowledgement}

The authors are grateful to the Research Centre M S Ramaiah Institute of Technology, Atria Institute of Technology, Vivekananda Institute of Technology, for all the support and also the financial support from VTU research scheme project.

\section{References}

[1] Bejan A. and Poulikakos D.(1984): "The non-Darcy regime for vertical boundary layer natural convection in a porous medium." Internl. J. H eat M ass Transfer, vol.27, pp.717-722. 
[2] Benanati R.F. and Brosilow C.B. (1962): “Void fraction distribution in beds of spheres."AI Che J., vol.8,pp.359-361.

[3] Chandrasekhara B.C. and Namboodiri P.M.S.(1985): “Influence of variable permeability on combined vertical surfaces in porous medium." Internl. J. H eat M ass Transfer, vol.28,pp.199-206.

[4] Chen K.S. and Ho J.R.(1988): "Effects of flow inertia on vertical natural convection in saturated porous media." Internl. J. H eat M ass Transfer ,vol.29,pp.753-759.

[5] Cheng P.(1978): "Heat transfer in geothermal systems," Adv. H eat Transfer,vol.4,pp.1-105.

[6] Cheng P. and Minkowycz W.J.(1977): “Free convection about a vertical plate embedded in a porous medium with application to heat transfer from a dike."J. G eophys. Res.vol.82, pp.2040-2044.

[7] Hady F.M., Bakier A.K. and Gorla R.S.R.(1996): “Mixed convection boundary layer flow on a continuous flat plate with variable viscosity." H eat mass Transfer, vol.31, pp.169-172.

[8] Hassanien I.A., Bakier A.Y. and Gorla R.S.R.(1998): “Effects of thermal dispersion and stratification on non-Darcy mixed convection from a vertical plate in a porous medium." $H$ eat $M$ ass Transfer,vol.34,pp.209-212.

[9] Hong J.T., Yamada Y. and Tien C.L.(1987): “Effect of nonDarcian and non-uniform porosity on vertical plate natural convection in porous media." Internl. J. H eat M ass Transfer, vol.109, pp.356-382.

[10] Hsieh J.C., Chen T.S. and Armaly B.F.(1993): “Non-similarity solutions for mixed convection from vertical surfaces in porous medium with variable surface temperature or heat flux." Internl. J. H eat M ass Transfer, vol.38,N o.4, pp.1485-1493.

[11] Hung C.I. and Chen C.B.(1997): “N on-Darcy free convection in a thermally stratified porous medium along a vertical plate with variable heat flux." H eat and M ass Transfer, vol.33,pp.101-107.

[12] Kumari M., Pop I.and Nath G.(1990): “Non - Darcian effects on forced convection heat transfer over a flat plate in a highly porous medium." Acta M echanica, vol.84, pp.201-207. 
[13] Lai F.C. and Ku lacki F.A. (1987): "N on-Darcy convection from horizontal impermeable surface in saturated porous medium." Internl. J. H eat M ass Transfer, vol.30, pp2189-2192.

[14] Lai F.C. and Kulacki F.A.(1991): "Non - Darcy mixed convection along a vertical wall in a saturated porous medium." Internl.J.H eat M ass Transfer,vol.113, pp.252-255.

[15] Merkin J.H.(1980): "Mixed convection boundary layer flow on a vertical surface in a saturated porous medium." J. of Engineering $M$ athematics,vol.14,N o.4,pp.301-313.

[16] Mohammadein A.A. and El-Shaer N.A. (2004): “Influence of variable permeability on combined free and forced convection flow past a semi - infinite vertical plate in a saturated porous medium." H eat M ass Transfer, vol.40, pp.341-346.

[17] Nakayama A.and Koyama H. (1987): "Effect of thermal stratification on free convection within a porous medium." J. Thermophysics H eat Transfer, vol.1,pp.282-285.

[18] Nield D.A. and Bejan A.(1999): Convection in porous media. Springer verlag.

[19] Plump O.A. and Huenefeld J.C. (1981): “Non - Darcy natural convection from heated surfaces in saturated porous media." Internl. J. H eat M ass Transfer, vol.24, pp.765-768.

[20] Schwartz C.E. and Smith J.M. (1953): "Flow distribution in packed beds." Ind. Eng. Chem., vol.45, pp.1209-1218.

[21] Vajravelu K. (2001): "Viscous flow over a nonlinear stretching sheet." A pplied M ath. Computation, vol.124, pp.281-288. 